\title{
A new PWM method for a 3-phase 4-leg inverter based on the injection of the opposite median reference voltage
}

\author{
Abdelkader Bouarfa, Maurice Fadel \\ LAPLACE \\ Université de Toulouse, CNRS, INPT, UPS \\ Toulouse, France \\ \{bouarfa,fadel\}@laplace.univ-tlse.fr
}

\author{
Marc Bodson \\ Electrical and Computer Engineering \\ University of Utah \\ Salt Lake City, Utah, USA \\ bodson@eng.utah.edu
}

\begin{abstract}
In this paper, a new carrier-based pulse-width modulation (PWM) technique for a voltage source inverter (VSI) is presented. The new modulation law is obtained using a particular configuration of a control allocation technique based on a perswitching-state formulation of the 4-leg 2-level inverter. The resulting modulation law is equivalent to the injection of the opposite of the median reference voltage as a zero-sequence voltage. This particular solution produces a smooth transition from a continuous modulation mode at low modulation depth to a discontinuous modulation mode at high modulation depth. This hybrid solution makes it possible to focus on the THD for low output voltages and reduce losses for higher voltages.
\end{abstract}

Keywords-carrier-based PWM; 4-leg 2-level inverter; VSI; continuous-discontinuous PWM transition method;

\section{INTRODUCTION}

A vast number of modulation laws have been proposed for the output voltage control of inverters [1]-[2]. Some of them were identified for specific applications, bringing interesting properties suited to them. A popular class of modulation strategies, called fixed high-switching-frequency pulse-width modulation (PWM), rests on the "per-switchinginterval volt-second average" principle: the objective is to obtain the desired voltage in mean value over a given period sufficiently shorter than the fundamental period.

The first PWM technique proposed for the 3-leg 2-level inverter is the sinusoidal pulse-width modulation (SPWM), which is a carrier-based PWM (CBPWM) method [1]-[2]. With CBPWM, duty cycles are determined by comparison between a low-frequency modulating signal and a high-frequency carrier. For SPWM, the modulating signal simply corresponds to the desired fundamental sinewave voltage. With SPWM, however, the output voltage amplitude is limited to half of the DC bus voltage, in linear mode.

Neutral point voltage control exploits the degree of freedom available to obtain the desired load voltages while adding useful properties, such as a $15 \%$ extension of the linear range of the inverter. In this manner, linear operation up to $1 / \sqrt{3} \cdot E_{D C}$ is enabled. Among PWM methods offering this property, space vector modulation with equally used zero vectors [2]-[3]
(SVM), or regular-sampled symmetric PWM [4] (RSPWM) with 1/6 third harmonic injection [5], [6] (THIPWM1/6), are popular for their superior harmonic performance. Discontinuous PWM (DPWM) schemes allow a sensible reduction of switching losses and mean switching frequency [7]. For CBPWM methods, the neutral point voltage control corresponds to the injection of a zero-sequence voltage, which is normally a harmonic 3 (or an odd number multiple of 3). For space vector based strategies, the neutral point voltage control endues from the use of the two zero vectors of the inverter.

The degree of freedom offered by the control of the neutral point voltage can be included in an algebraic formulation of the operation of the inverter. Then, it is advantageous to use this degree of freedom in an optimized manner in real-time, to achieve the desired performance. Control allocation methods [8]-[10] provide a solution to this problem. They concern control problems that are over-determined, as in flight control or marine applications. Operation is optimized while accounting for ranges of motion. Consequently, the solutions resulting from control allocation methods yield a maximal extension of the linearity range. Details about the control allocation method used will be discussed in a future paper.

A focus on a particular configuration of the control allocation algorithm leads to a new PWM method giving an interesting property: it yields a continuous transition between continuous and discontinuous modulation schemes as a function of the reference amplitude. This new method can be interpreted as the injection of the opposite of the median voltage as a zerosequence voltage. Thus, we call this new modulation law Opposite Median-Voltage Injection PWM (OMIPWM).

In the case of an unbalanced load, the use of a 4-leg 2-level inverter gives a direct control of the neutral potential, which facilitates the injection of zero-sequence voltages through the fourth leg. Also, the control allocation method which is the origin of OMIPWM was constructed for the 4-leg 2-level inverter. For these reasons, the properties of OMIPWM will be explained and illustrated using a 4-leg inverter. Nevertheless, OMIPWM works also with the 3-leg inverter under the "threephase balanced voltages and load" assumption, as the neutral potential will correspond to the mean of the reference voltages. At the end of this paper, simulation results are given with a 
performance comparison between OMIPWM, SVM, DPWMmin and DPWMmax [1], [2], [7].

\section{FOUR-LEG TWO-LEVEL INVERTER}

The 4-leg 2-level inverter is used for several applications like active filtering, decentralized generation, four-wire systems, polyphase, nonlinear or unbalanced loads, etc. The fourth leg allows one to control the neutral point voltage and, as a result, the independent control of the load voltages. The neutral point connection can also handle eventual unbalanced currents.

\section{A. Connection with the load}

Fig. 1 illustrates the inverter, star-connected to a three-phase load with per-phase impedances $Z_{A}, Z_{B}, Z_{C}$. $E_{D C}$ denotes the DC bus voltage that can be obtained from batteries or from the three-phase network through a rectifier. The load can be unbalanced or nonlinear. The fourth-leg $(N)$ is connected to the neutral point of the load in order to control the neutral voltage. Here, the impedance $Z_{N}$ on the neutral wire is neglected.

\section{B. Switching cells}

One considers a switching cell as an association of two functional switches. Each leg of the inverter is comprised of one switching cell. Dead times are neglected: the binary states of the two switches of a leg are complementary all the time.

Under this assumption, one defines the state of a switching cell as the state of the corresponding upper switch. For $K \in\{A, B, C, N\}$, the binary state $S_{K}$ of the upper switch of the leg- $K$ switching cell is 0 when the switch is off and 1 when the switch is on.

\section{Output voltages, load voltages}

The output voltages (referred to the ground $G$ ) are given from the switching cell states by a linear transformation:

$$
\begin{aligned}
& \forall K \in\{A, B, C, N\}, \quad V_{K G}=E_{D C} S_{K} \\
& \forall K \in\{A, B, C, N\}, \quad V_{K G} \in\left\{0, E_{D C}\right\}
\end{aligned}
$$

The three-phase load voltages are equal to the difference between the three first legs' output voltages and the fourth leg voltage. As the 4-leg inverter can only give three independent load voltages, it might be interesting to define for each of the three first legs a relative switching state $S_{K N}$ :

$$
\forall K \in\{A, B, C\}, \quad S_{K N}=S_{K}-S_{N}
$$

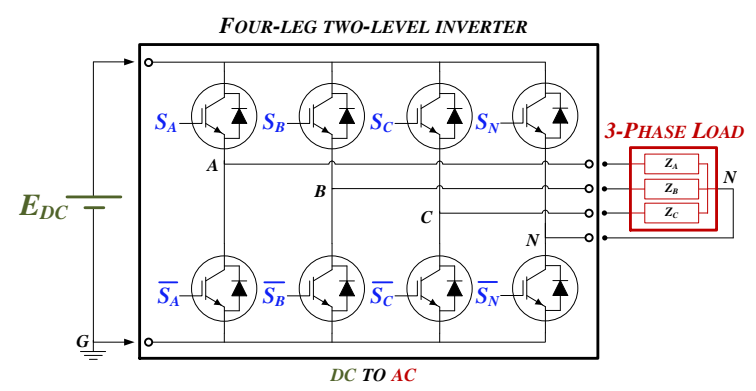

Fig. 1. Illustration of the 4-leg 2-level inverter connected to a three-phase load with impedances $Z_{A}, Z_{B}, Z_{C}$.

$$
\forall K \in\{A, B, C\}, \quad S_{K N} \in\{-1,0,1\}
$$

Then, the load voltages are given by

$$
\begin{gathered}
\forall K \in\{A, B, C\}, \quad V_{K N}=E_{D C} S_{K N} \\
\forall K \in\{A, B, C\}, \quad V_{K N} \in\left\{-E_{D C}, 0, E_{D C}\right\}
\end{gathered}
$$

\section{Pulse-Width Modulation}

Note $\langle U\rangle_{T s}$ the mean value of any unspecified quantity $U$ over the unspecified period $T_{S}$. Thus, for each switching cell $K$, one defines the duty cycle $D_{K}$ of the upper switch as the mean value of the switching state $S_{K}$ over the switching period $T_{S}$ :

$$
\begin{gathered}
\forall K \in\{A, B, C, N\}, \quad D_{K}=<S_{K}>_{T_{S}} \\
\forall K \in\{A, B, C, N\}, \quad D_{K} \in[0,1]
\end{gathered}
$$

By convention, the gating pulses will be centered into the switching period $T_{S}$, as illustrated on Fig. 2. Generally, there will be two switchings per switch over a switching period.

The mean values of the inverter output voltages over $T_{S}$ are given by the following expressions:

$$
\begin{gathered}
\forall K \in\{A, B, C, N\}, \quad<V_{K G}>_{T_{S}}=E_{D C} D_{K} \\
\forall K \in\{A, B, C\}, \quad<V_{K N}>_{T_{S}}=E_{D C}\left(D_{K}-D_{N}\right)
\end{gathered}
$$

and, by considering each leg of the inverter individually:

$$
\begin{gathered}
\forall K \in\{A, B, C, N\}, \quad<V_{K G}>_{T_{S}} \in\left[0, E_{D C}\right] \\
\forall K \in\{A, B, C\}, \quad<V_{K N}>_{T_{S}} \in\left[-E_{D C}, E_{D C}\right]
\end{gathered}
$$

The mean value notation will be subsequently removed for voltage quantities, since mean values are used for computations.

\section{A NEW PWM METHOD ALLOWING SMOOTH TRANSITION BETWEEN CONTINUOUS AND DISCONTINOUS PWM SCHEMES}

\section{A. A control allocation method for the 4-leg 2-level inverter} using a per-switch functional formulation

\section{1) Per-switch functional formulation}

The 4-leg 2-level inverter control problem can be formulated by considering its mathematical function. Under the assumptions mentioned above, for each switching period $T_{S}$, the objective is to find a set of duty cycles $D_{K}$ to obtain a reference vector $V_{\text {ref }}$ of load voltages $V_{K N r e f}$ :

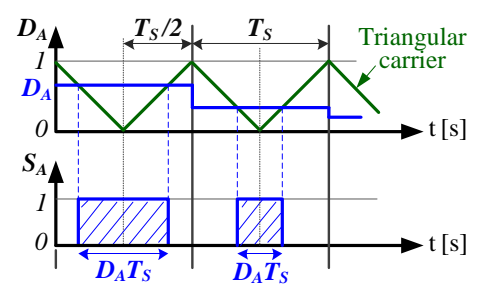

Fig. 2. Illustration of the realization of gating pulse on switch $\mathrm{A}$ of width $D_{A} \cdot T_{S}$ and centered over the switching period $T_{S}$. 
$\forall K \in\{A, B, C\}, \quad V_{K N_{r e f}}=<V_{K N}>_{T_{S}}=E_{D C}\left(D_{K}-D_{N}\right)$

Define $\Delta D_{r e f}$, the scaled reference vector, as follows:

$$
\Delta D_{r e f}=\frac{V_{r e f}}{E_{D C}}=\left(\begin{array}{c}
D_{A N r e f} \\
D_{B N r e f} \\
D_{C N \text { ref }}
\end{array}\right)
$$

$\Delta D_{\text {ref }}$ is similar to the difference of duty cycles between the three first legs and the fourth one.

From the expressions of the load voltages as functions of the switching states, one finds the matrix functional formulation:

$$
\begin{gathered}
\Delta D_{r e f}=\left(\begin{array}{llll}
1 & 0 & 0 & -1 \\
0 & 1 & 0 & -1 \\
0 & 0 & 1 & -1
\end{array}\right) \cdot\left(\begin{array}{l}
D_{A} \\
D_{B} \\
D_{C} \\
D_{N}
\end{array}\right) \\
\forall K \in\{A, B, C, N\}, 0 \leq D_{K} \leq 1
\end{gathered}
$$

Without constrains, the system would have an infinite number of solutions. The constraints (16) expressed on the duty cycles are an additional difficulty for solving the problem.

\section{2) Control allocation methods}

Control allocation methods are an interesting solution for handling degree of freedoms available for the control of the inverter [10]. Control allocation theory was developed as a solution for overdetermined problems (that can have more than one solution) and whose decision variables are subjected to constraints [9]. Flight control is a primary field of application [8].

Control allocation methodology rests on three points:

- one wants to impose reference values to some quantities to be controlled;

- one has resources that are generally redundant;

- there are constraints mainly linked to resources, or actuators' ranges of motion, that have to be respected in order to obtain the desired quantities.

The main objective is to realize an automatic distribution of the references to the available resources while respecting the constraints, and to do this at each control instant. Most of the time, difficulties come from the constraints that reduce the set of solutions, although these solutions may not be unique, even with constraints.

For the 4-leg 2-level inverter, the references are the desired load voltages, the constraints are linked to the limited time of the switching period [10], and the switching cells are the resources.

For the problem (15)-(16), one searches a solution that is feasible, i.e., respects the constraints on the duty cycles, and results in the desired load voltages. There might be zero, one, or an infinity of such solutions. An interesting proposition is to find a solution for the problem in an optimized manner, by formulating one or more performance or control criteria. The on-line computation cost is high, but the performances of mod- ern computers or digital signal processors increasingly allow one to overcome this issue.

\section{B. OMIPWM : A particular configuration of a control allocation technique dedicated to the 4-leg 2-level inverter}

The modulation method introduced in this paper comes from a specific choice of optimization criterion. More details about the general control allocation method will be given in a future paper. Here, the focus is placed on the original properties of the specific case. The result will be interpreted as a CBPWM method, with triangular intersection.

Fig. 3 shows the corresponding control diagram. Under the assumptions formulated in this paper, the duty cycles can be obtained by choosing $D_{N}$ while respecting the correct distances between the duty cycles expressed by $\Delta D_{\text {ref: }}$ :

$$
\left(\begin{array}{l}
D_{A} \\
D_{B} \\
D_{C}
\end{array}\right)=\Delta D_{r e f}+D_{N}
$$

The injection of the zero-sequence voltage can be represented by the waveform of the fourth duty cycle $D_{N}$ through the quantity $D_{Z S V}$ :

$$
D_{N}=\frac{1}{2}+D_{Z S V}
$$

In the case of a three-phase balanced system, it corresponds to the well-known form of any modulating signal:

$$
\left(\begin{array}{c}
D_{A} \\
D_{B} \\
D_{C}
\end{array}\right)=\frac{1}{2}+\frac{A_{r e f}}{E_{D C}}\left(\begin{array}{c}
\sin (\omega t) \\
\sin (\omega t-2 \pi / 3) \\
\sin (\omega t+2 \pi / 3)
\end{array}\right)+D_{Z S V}
$$

where $A_{\text {ref }}$ is the peak amplitude of the three-phase reference voltage system.

To ensure that all duty cycles are between 0 and 1 , the following bounds must be satisfied:

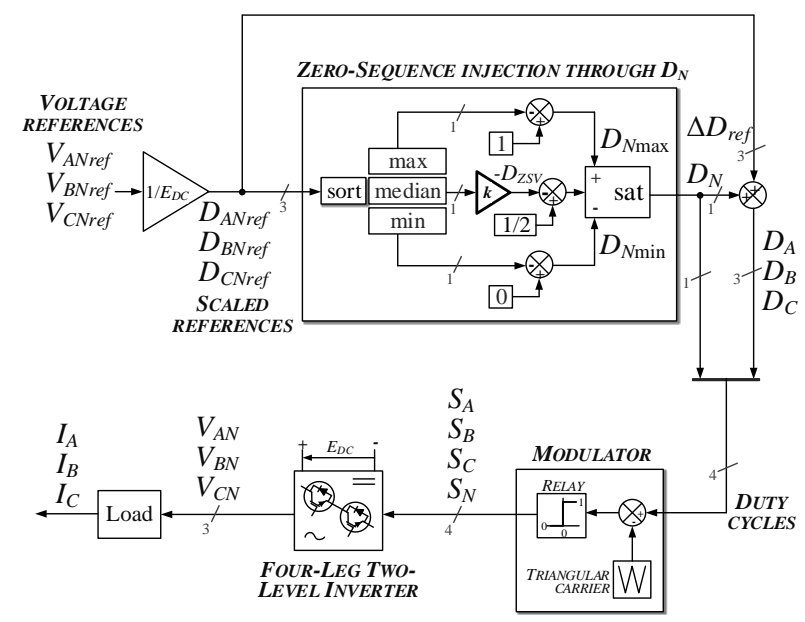

Fig. 3. Proposed control diagram, based on the ordering of the reduced reference voltages. The parameter $k$ quantifies the injection of the opposite of the median voltage as a zero-sequence. 


$$
\begin{gathered}
D_{N_{\text {min }}}=-\min \Delta D_{r e f} \\
D_{N_{\text {max }}}=1-\max \Delta D_{r e f} \\
D_{N_{\text {min }}} \leq D_{N} \leq D_{N_{\text {max }}}
\end{gathered}
$$

The quantities $D_{N \min }$ and $D_{N \max }$ specify the available margin for injecting zero-signals while ensuring feasible duty cycles. To be rigorous, these bounds should be further limited to the interval from 0 to 1 , but this step is omitted for simplicity. If $D_{N}$ stays between $D_{N \min }$ and $D_{N \max }$, the desired load voltages can be achieved.

The higher the reference amplitude is, the smaller the domain described by these two bounds becomes. In the case of a three-phase balanced system, the linearity range is extended to $1 / \sqrt{ } 3 \cdot E_{D C}$, i.e., almost $58 \%$ of the DC bus voltage. When $D_{N}=$ $D_{N \min }$, one leg will be clamped to the negative pole of the DC bus (ground) and, for a whole fundamental period, this choice corresponds to a modulation of type DPWMmin. When $D_{N}=$ $D_{N \max }$, one leg will be clamped to the positive pole of the DC bus and, for a whole fundamental period, this choice corresponds to a modulation of type DPWMmax.

The particularity of OMIPWM lies in the combined effects of the saturation of $D_{N}$ by $D_{N \min }$ and $D_{N \max }$ and by the use of the opposite of the median reference voltage as a zero-sequence. Actually, the median reference voltage is an advantageous and quite simple choice of zero-sequence voltage, as it is completely composed of harmonics that are multiple of three and is directly deduced from the three reference voltages. It has been shown that the regular-sampled symmetric PWM with injection of the half of the median reference voltage is equivalent to SVM [11]. This result can be also deduced from other studies [12]-[13]. In [14], the given formulas for SVM can be expressed with our notation as follows:

$$
\begin{aligned}
D_{N S V M} & =\frac{D_{N_{\min }}+D_{N_{\max }}}{2} \\
& =\frac{1}{2}-\frac{\max \Delta D_{r e f}+\min \Delta D_{r e f}}{2}
\end{aligned}
$$

Under the "three-phase reference voltages" assumption, the sum of the reference voltage is null, and the SVM corresponding zero-sequence signal is

$$
D_{\text {ZSVSVM }}=\frac{1}{2} \cdot \operatorname{med} \Delta D_{\text {ref }}
$$

A crucial point is the fact that (23) means that $D_{N S V M}$ is the mean (or the center) of $D_{N \min }$ and $D_{N \max }$. Thus, discontinuous modulation is naturally avoided when using (23) and when $D_{N \min }$ is inferior to $D_{N \max }$.

Contrary to this property, the new zero-sequence signal proposed by OMIPWM is still based on the median voltage, but through the choice:

$$
D_{\text {ZSV OMIPWM }}=-\operatorname{med} \Delta D_{r e f}
$$

As the reference amplitude increases, $D_{N}$ will be more inclined to reach the bounds $D_{N \min }$ and $D_{N \max }$ and, at that moment, dis- continuous modulation appears with increasing discontinuous periods. OMIPWM is a simple method that automatically ensures the transition from a continuous to a discontinuous modulation scheme as a function of the reference amplitude. When in discontinuous modulation mode, all switches will be once clamped to the DC bus and once to the ground over a fundamental period, as it will be shown in the next section.

An improvement of the method can be obtained by the introduction of an injection factor $k$,

$$
D_{\text {ZSVOMIPWM }}=-k \cdot \operatorname{med} \Delta D_{r e f}
$$

that modulates the transition effect and causes a transition from continuous to discontinuous modulation at an adjustable level. This will be also illustrated later.

The determination of $D_{N \min }, D_{N \max }$ and the zero-sequence signal $D_{Z S V}$, is obtained from an ordering of the three reference voltages, needing 2 comparisons. Then, the duty cycles are determined by adding the chosen $D_{N}$ to the scaled reference vector $\Delta D_{\text {ref. }}$. Finally, the gating signals are given by the modulator block.

\section{SimUlation RESUlts}

In this section, OMIPWM is evaluated in simulation and compared with SVM, DPWMmin and DPWMmax through different criteria. Simulations were carried out in a MATLABSimulink environment. The main simulation parameters are summarized in Table I. All simulations were performed under the "three-phase balanced voltages and load" assumption for simplicity. Therefore, the same results can be obtained with the three-leg inverter.

Duty cycle waveforms are shown on Fig. 4. The reference amplitude value increases from left (a) to right (c) until the limit of $1 / \sqrt{ } 3$ is reached. The representation of duty cycles as functions of the variable $\theta=2 \pi \mathrm{ft}$, $\mathrm{t}$ being time, allows abstraction of the fundamental frequency $f$. A whole fundamental period is illustrated. OMIPWM is configured with $k=1$.

The zero-sequence signals can be deduced from $D_{N}$ waveforms on the upper curves $(x 1)$ of Fig. 4 . On these figures, the extremal bold curves correspond to the bounds specified by $D_{N \min }$ and $D_{N \max }$. The respect of these limits ensures that the resulting duty cycles $D_{A}, D_{B}, D_{C}$ are between 0 and 1 . On Fig. 4 (a1), the bounds are not reached and $D_{N \text { OMIPWM }}$ corresponds to the opposite of the median reference voltage. Contrary to SVM, where the zero-sequence voltage is the half of the positive median voltage, for OMIPWM, the distance between $D_{N}$ OMIPWM and the bounds $D_{N \min }$ and $D_{N \max }$ at the points $\theta=\pi / 6+$ $n^{*} \pi / 3, n=0 . .5$, is small and decreases when the reference amplitude increases. This is the reason why saturation appears on

TABLE I. SIMULATION PARAMETERS

\begin{tabular}{ccc}
\hline \hline Symbol & Meaning & Values \\
\hline$E_{D C}$ & DC bus voltage & $400 \mathrm{~V}$ \\
$f_{s}$ & Switching frequency & $10 \mathrm{kHz}$ \\
$f$ & Load current frequency & $50 \mathrm{~Hz}$ \\
$R$ & Load resistance & $0.5 \Omega$ \\
$L$ & Load inductance & $10 \mathrm{mH}$ \\
\hline \hline
\end{tabular}




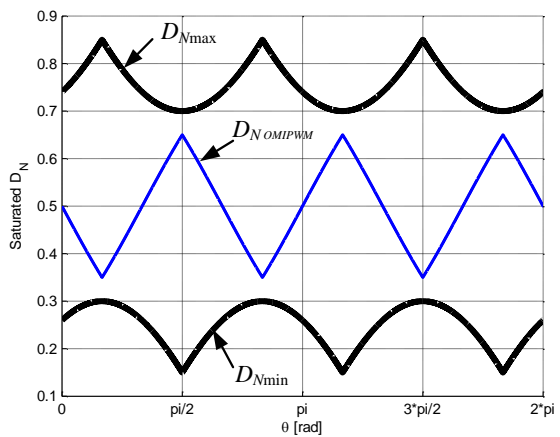

(a1)

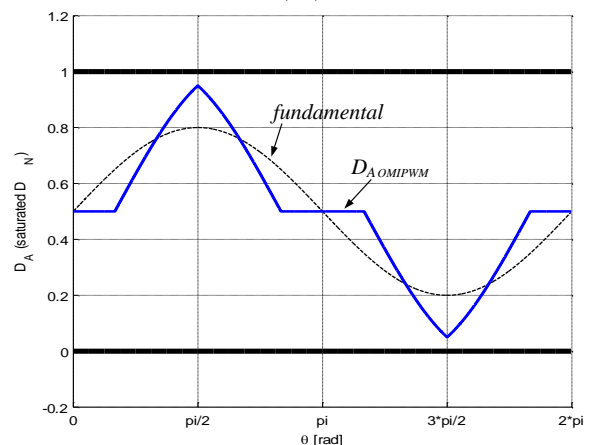

(a2)

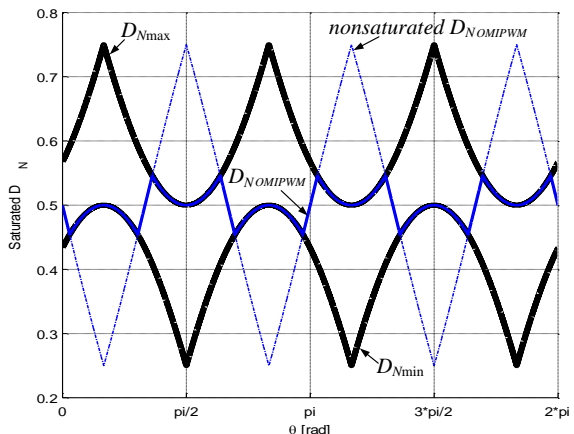

(b1)

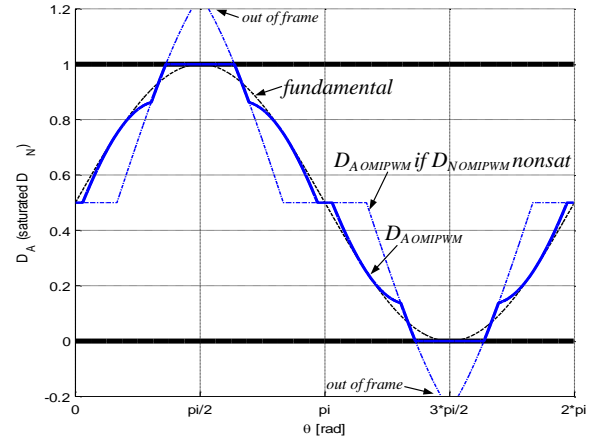

(b2)

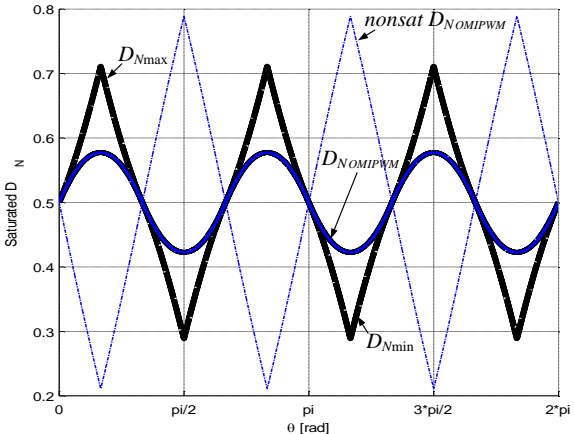

(c1)

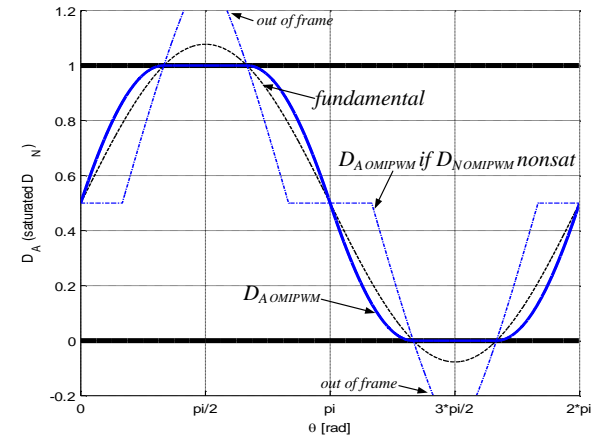

(c2)

Fig. 4. Waveforms of $D_{N}\left(1\right.$, up) and $D_{A}\left(2\right.$, down) from OMIPWM for several reference amplitudes. (a) $V_{\text {ref }}=0.3 E_{D C}$. (b) $V_{r e f}=0.5 E_{D C}$. (c) $V_{r e f}=1 / \sqrt{3} E_{D C}$.

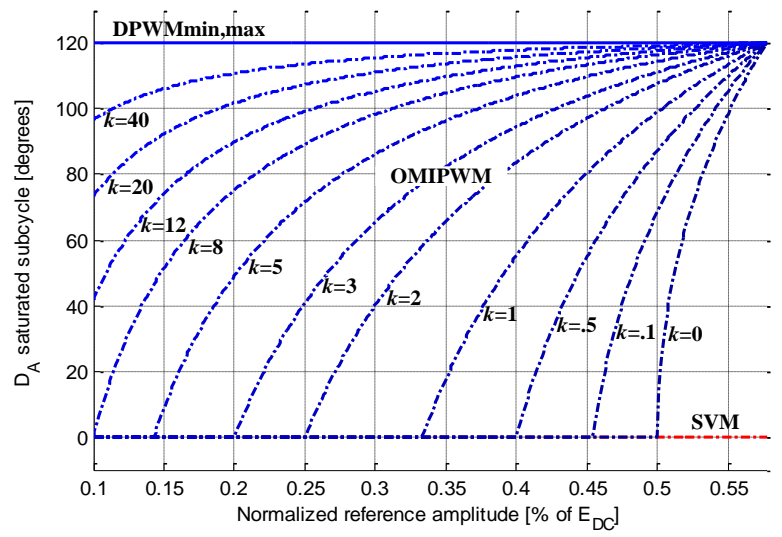

Fig. 5. Total clamping duration (in degrees) per switch over a fundamental period as a function of the reference amplitude.

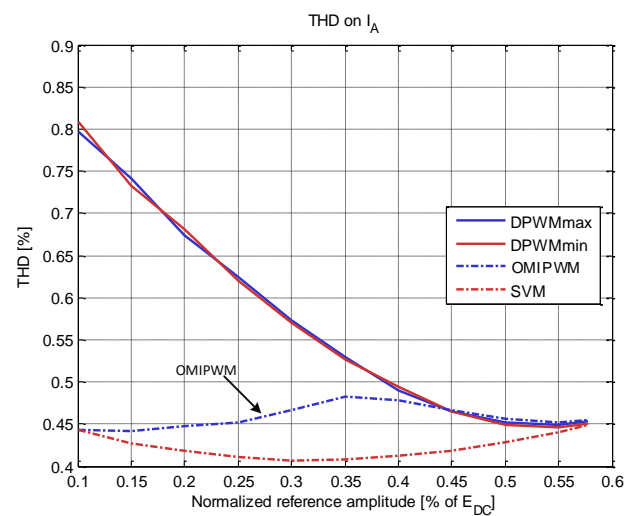

Fig. 7. Total harmonic distortion on phase A load current as a function of the reference amplitude $(k=1)$.

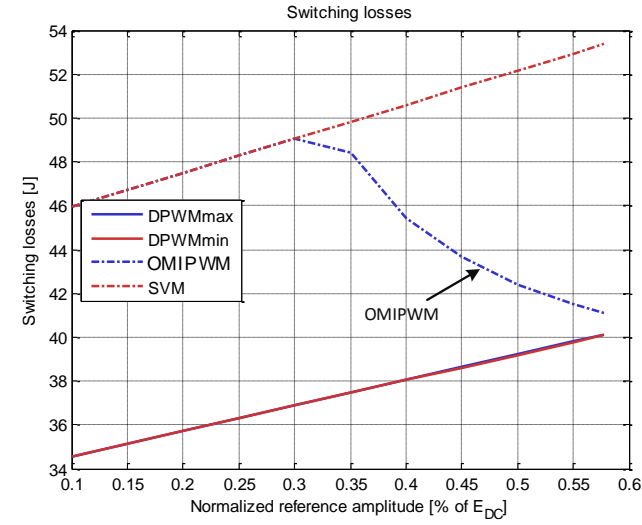

Fig. 6. Total switching losses of the inverter as functions of the reference amplitude $(k=1)$.

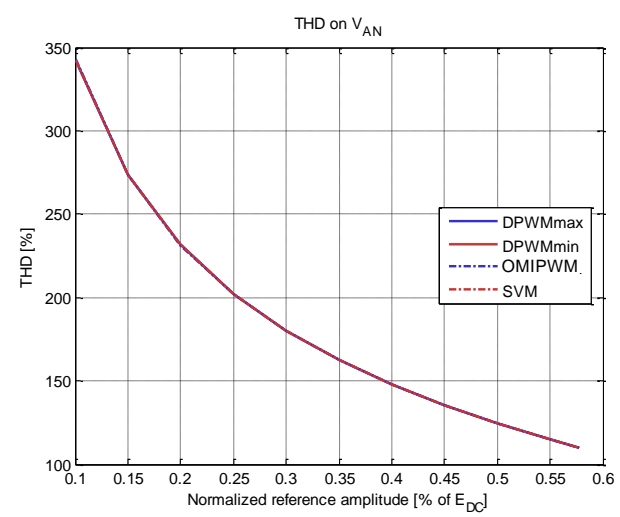

Fig. 8. Total harmonic distortion on phase A load voltage as a function of the reference amplitude $(k=1)$. 
Fig. 4 (b1). The typical quasi-triangular waveform of the unsaturated $D_{\text {NOMIPWM }}$ is seen as the dash-dotted blue curve, which crosses the bounds and causes the appearance of discontinuous modulation scheme. Over the fundamental period, the parts of $D_{N \text { OMIPWM }}$ saturated by $D_{N \min }$ and $D_{N \max }$ correspond to periods where a leg is continuously clamped to the DC bus or to the ground, as observed on the waveform of $D_{A}$ on Fig. 4 (b2). As a result of this saturation, the waveform of $D_{A}$ OMIPWM is modified, compared to Fig. 4 (a2), in order to obtain the correct fundamental waveform (black dashed line).

At the maximum voltage of $1 / \sqrt{ } 3 \cdot E_{D C}$ permitting linearity, OMIPWM is equivalent to DPWM1 scheme [1], [7], [15], resulting in an alternation of $60^{\circ}$-width periods between DPWMmin and DPWMmax.

Fig. 5 illustrates clearly the smooth transitional effect of OMIPWM. For DPWM schemes, the total clamping period over a fundamental period corresponds to $120^{\circ}$ (per switch). For OMIPWM, from a certain amplitude value, this period becomes non null and increases until the maximum value of $120^{\circ}$ at the maximum reduced reference amplitude of $1 / \sqrt{3}$. The injection coefficient $k$ makes the transition more or less abrupt. This result is obtained through a transition that starts earlier and ends later.

The transition effect also has an effect on the switching losses, shown on Fig. 6. As the evaluation of switching losses depends on the load characteristics and the modulation method, we focus our attention only on the general trends of the curves. Two quasi-parallel lines, one higher than the other, describe the evolution of the switching losses, respectively, for SVM and for DPWMmin, DPWMmax. The trend for OMIPWM is remarkable since the curve falls from the SVM curve to the DPWM curves as a function of the amplitude.

Fig. 7 and Fig. 8 show the evolution of the total harmonic distortion (THD) on currents and on voltages, respectively. THD was evaluated thanks to the thd function of the Signal Processing Toolbox available in the MATLAB environment. Fig. 8 shows that THD trends on voltages are very close for all methods, but differences appear for THD's on currents, see Fig. 7, meaning differences on the spectral distribution. OMIPWM current THD trend is singular again. At low amplitude, THD on the currents is higher than SVM but fairly close compared to DPWM ones. As the amplitude increases, THD on currents for OMIPWM converges to DPWM THD's, which have decreased and are closer to the SVM's THD.

In conclusion, OMIPWM gives better harmonic performance at low voltage amplitude and lower switching losses at high voltage amplitude, meaning an advantageous compromise between continuous and discontinuous modulation properties as a function of the reference voltage amplitude. Thus, it might be interesting to consider this method for supplying a threephase machine. At low speeds (low voltages, low modulation depths), one has an interest in favoring THD in order to limit torque ripple. For high speeds, ripple is better filtered and it is more interesting to reduce switching losses.

\section{CONCLUSION}

A new carrier-based PWM technique for a voltage source inverter is presented in this paper. It uses the opposite of the me- dian reference voltage as a zero-sequence voltage, which yielded the acronym OMIPWM. The PWM method has the same linear range as the SVM, while offering a new, automatic transition between continuous and discontinuous modulation schemes. The period of transition from the continuous to discontinuous can be adjusted by setting a single parameter. The method was obtained using a specific configuration of a control allocation method for the 4-leg 2-level inverter that will be discussed in a future paper.

Results were obtained in simulations and various waveforms were shown. OMIPWM gives a new compromise between the good harmonic performance of continuous PWM methods like SVM and the reduced switching losses of discontinuous PWM methods like DPWMmin and DPWMmax.

\section{REFERENCES}

[1] D. G. Holmes and T. A. Lipo, Pulse Width Modulation for Power Converters: princples and practice, New York: IEEE/WileyInterscience, 2003.

[2] J. Holtz, "Pulsewidth Modulation for Electronic Power Conversion," Proc. IEEE, vol. 82, no. 8, pp. 1194-1214, Aug. 1994.

[3] H. W. Van der Broeck, H.-C. Skudelny and G. V. Stanke, "Analysis and Realization of a Pulsewidth Modulator Based on Voltage Space Vectors," IEEE Trans. on Industry Applications, vol. 24, no. 1, pp. 142 150, Jan.-Feb. 1988.

[4] S. R. Bowes, "New sinusoidal pulsewidth-modulated invertor," Proc. Inst. Elect. Eng., vol. 122, pp. 1279-1285, Nov. 1975.

[5] G. Buja and G. Indri, "Improvement of Pulse Width Modulation Techniques," Archiv für Elektrotechnik, vol. 57, pp. 281-289, 1975.

[6] J. A. Houldsworth and D. A. Grant, "The Use of Harmonic Distortion to Increase the Output Voltage of a Three-Phase PWM Inverter," IEEE Trans. on Industrial Applications, vol. IA-20, no. 5, pp. 1224-1228, Sept.-Oct. 1984.

[7] A. M. Hava, R. J. Kerkman and T. A. Lipo, "A High-Performance Generalized Discontinuous PWM Algorithm," IEEE Trans. on Indus. Appl., vol. 34, no. 5, pp.1059-1071, Sept.-Oct. 1998.

[8] M. Bodson, "Evaluation of Optimization Methods for Control Allocation," Journal of Guidance, Control and Dynamics, vol. 25, no. 4, pp. 703-711, July-Aug. 2002.

[9] T. A. Johansen and T. I. Fossen, "Control Allocation-A survey," Automatica, vol. 49, pp. 1087-1103, 2013.

[10] A. Bouarfa, M. Fadel, M. Bodson and J. Lin, "A New Control Allocation Method for Power Converters and its Application to the Four-Leg Two-Level Inverter," in IEEE $23^{\text {rd }}$ Mediterranean Conf. Control and Automation (MED 2015), Torremolinos, Spain, pp. 1020 1026, June 2015.

[11] P. F. Seixas, "Commande numérique d'une machine synchrone autopilotée," Ph.D. thesis, INPT, Toulouse, France, 1988.

[12] D. W. Chung, J. S. Kim and S. K. Sul, "Unified voltage modulation technique for real time three-phase power conversion," in Proc. Conf. Rec. IAS, pp. 921-926, 1996.

[13] C. B. Jacobina, A. M. N. Lima, E. R. C. da Silva, R. N. C. Alves and P. F. Seixas, "Digital Scalar Pulse-Width Modulation: A Simple Approach to Introduce Non-Sinusoidal Modulating Waveforms," IEEE Trans. Power Electronics, vol. 16, no. 3, pp. 351-359, May 2001.

[14] X. Li, Z. Deng, Z. Chen and Q. Fei, "Analysis and Simplification of Three-Dimensional Space Vector PWM for Three-Phase Four-Leg Inverters," IEEE Trans. Industrial Electronics, vol. 58, no. 2, pp. 450 464, Feb. 2011

[15] M. Depenbrock, "Pulse width control of a 3-phase inverter with nonsinusoidal phase voltage," in Conf. Rec. IEEE Int. Semiconductor Power Conversion Conf., pp. 399-403, 1977. 